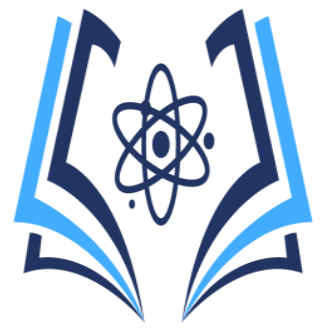

Factores de riesgo psicosocial en sanitarios que laboran en unidades de diálisis en Portoviejo y Manta, 2019

\author{
Psychosocial risk factors in sanitary workers working in dialysis \\ units in Portoviejo and Manta, 2019
}

\title{
Factores de riesgo psicosocial
}

\begin{abstract}
Simón Moreira Cuenca. MgSc. ${ }^{1}$
José Barreto Loor. Dr. ${ }^{2}$

Escobar Segovia Kenny. MgSc. ${ }^{3}$

${ }^{1}$ Magister en Seguridad y Salud Ocupacional, Universidad Particular de Especialidades Espíritu Santo, Sanborondon, Guayas, Ecuador, smoreirac@uees.edu.ec

${ }^{2}$ Especialista En Orientación Familiar Integral, Universidad Técnica De Manabí, Portoviejo - Manabí Ecuador, fernando1984_loor@live.com ORCID ID: 0000-0002-3293-2270

${ }^{3}$ Magister en Gestión de la Productividad y la calidad, Escuela Politécnica Superior Del Litoral, Guayaquil Guayas - Ecuador. kescobar@espol.edu.ec ORCID ID: 0000-0003-1278-7640
\end{abstract}

Contacto: smoreirac@uees.edu.ec

\section{Resumen:}

El objetivo principal de este estudio es analizar los factores psicosociales y el nivel de riesgo a los que están expuestos el personal salubrista que labora en centros de diálisis de Portoviejo y Manta en el año 2019. La metodología empleada en el presente estudio es de tipo cuantitativa - descriptiva, de corte transversal, cuyo fin reside en identificar los factores de riesgo psicosocial entre los grupos de profesionales salubristas que laboran como cuidadores directos en Unidades de Hemodiálisis. Se estudiaron y tomaron datos en tres centros de diálisis en las ciudades de Portoviejo y Manta. En función al objeto de estudio se empleó como instrumento de recolección de datos el Cuestionario FPSICO 3.1, instrumento validado y ampliamente utilizado en otros estudios. Posteriormente, se realizaron entrevistas individuales con la finalidad de conocer datos socio-laborales y medidas asociativas con base a

\section{Summary:}

The main objective of this study is to analyze the psychosocial factors and the level of risk to which los factores psicosociales. Los profesionales que más se sienten afectados son los enfermeros, quienes perciben que la exigencia en el trabajo es alta (emocional o cognitiva), que se les demanda una extenuante concentración en su rol, adicional a que son supervisados de manera excesiva; lo que conlleva a un riesgo psicosocial latente con probables alteraciones en la salud mental. Se identificó que los factores psicosociales con riesgo más elevado que afectan a dichas instituciones y a los salubristas son la Carga de Trabajo (CT), las Demandas Psicológicas (DP), Participación/Supervisión y el Interés por el Trabajador o su Compensación, relacionados a una mayor exposición entre los profesionales de enfermería.

Palabras clave: Salud Mental, Factores psicosociales, Riesgo Laboral, Hemodiálisis.

the health personnel who work in dialysis centers in Portoviejo and Manta are exposed in 2019. The methodology used in this study is quantitative descriptive, cross-sectional, whose purpose is to 
identify psychosocial risk factors among groups of health professionals who work as direct caregivers in Hemodialysis Units. Data were studied and taken in three dialysis centers in the cities of Portoviejo and Manta. Depending on the object of study, the FPSICO Questionnaire 3.1 was used as a data collection instrument, a validated instrument and widely used in other studies. Subsequently, individual interviews were conducted in order to know socio-labor data and associative measures based on psychosocial factors. The professionals who are most affected are the nurses, who perceive

Keywords: Mental Health, Phsychosocial factors, Occupational Risks, Hemodialysis.

\section{Introducción:}

En Ecuador, la enfermedad renal crónica es la quinta causa de mortalidad prematura, con una afectación de 30.000 personas aproximadamente, de las cuales 11.000 son pacientes dializados (MSP, 2018), las unidades de hemodiálisis (HD) que atienden a este tipo de pacientes, son reguladas por el Ministerio de Salud Pública y entidades adscritas, designadas para garantizar la calidad de servicios de salud a nivel nacional. Este tipo de Centros Especializados en un III Nivel de atención, establecen como requerimiento fundamental la presencia de un equipo multidisciplinario, que incluya como cuidadores directos a enfermeros (1 por cada 7 pacientes), auxiliares de enfermería (1 por cada 14 pacientes) y médicos (1 por cada turno).

Este personal al interactuar constantemente con dichos pacientes, con un régimen de HD de 3 veces por semana, y al tener en sus manos el cuidado de la vida y salud de estas personas, se traduce en que un error en esta profesión podría devenir en consecuencias graves tanto para los pacientes como para los mismos trabajadores salubristas, Según Rudnicki (2006) el paciente en HD por lo general se rebela al tratamiento, no se compromete, se fastidia, se queja, se irrita fácilmente, es poco colaborador $\mathrm{y}$ sin iniciativa en sus propios cuidados, es regresivo en sus actitudes y comportamiento.

Diferentes estudios realizados en países de Latinoamérica demuestran interacciones entre el entorno laboral deficiente desde un punto de vista psicosocial y el estrés relacionado con las condiciones de trabajo, incrementando la probabilidad de sufrir un accidente laboral (OIT, 1999). La evidencia muestra claramente que diferentes factores de riesgo psicosocial como la
Vol. 3, Nro. 2, Publicado 2020-12-31

that the demand at work is high (emotional or cognitive), that they are demanded a strenuous concentration in their role, in addition to being overseen; which leads to a latent psychosocial risk with probable alterations in mental health. It was identified that the psychosocial factors with the highest risk affecting these institutions and health professionals are Workload (CT), Psychological Demands (DP), Participation / Supervision and Interest by the Worker or their Compensation, related to greater exposure among nursing professionals.

elevada carga de trabajo, las exigencias laborales, la escasa capacidad de decisión, el bajo criterio de las calificaciones, la falta de apoyo organizativo, los problemas con la dirección y los compañeros de trabajo y una importante monotonía en el trabajo están relacionados con la siniestralidad en el ámbito laboral (Mandujano \& Danae, 2018).

En el caso del personal de salud que labora en centros de hemodiálisis, se evidencian alteraciones psicológicas tales como problemas emocionales, ansiedad, estrés y responsabilidades en su rol, todos relacionados con su entorno laboral; teniendo como posibles consecuencias los accidentes laborales (pinchazos), alteraciones en la salud (física y mental), afectación en la relación personalpaciente, y/o violencia verbal o psicológica dirigida a los cuidadores directos por parte de los usuarios atendidos, problemática que ha sido ampliamente estudiada por diversos autores (Mandujano \& Danae, 2018). Los accidentes de trabajo se relacionan directamente con los factores de riesgo psicosocial y actividades sanitarias que se desarrollan dentro de la mente de cada profesional y dentro de las instalaciones de salud respectivamente (Castro, 2018).

Por otra parte, en un estudio comparativo se demostró que altas cargas de trabajo, falta de apoyo en el ambiente de trabajo, se traducen en un deterioro de la calidad de atención y de la satisfacción del usuario, y esto se asociaba con un aumento significativo del Síndrome de Burnout. (SB) en las enfermeras; una de cada tres enfermeras registradas presentaba Burnout, relacionados con factores como: edad, experiencia y no realizar turnos de noche (Flynn, Thomas-Hawkins, \& Clarke, 2009).

En Latinoamérica existen una serie de investigaciones y publicaciones sobre personal de enfermería que labora en unidades de HD, de los cuales destacamos un estudio realizado en Chile en 
Periodo. Julio - Diciembre 2020

el año 2012, que determinó que la sobrecarga laboral con jornadas de trabajo extensas, en una misma unidad de diálisis o servicio clínico, con dedicación exclusiva a la atención de pacientes y sin posibilidad de espacios de esparcimiento, son sin duda considerados como factores que aumentan el SB. Y si a lo anterior, se le agrega la carga afectiva a la que el profesional de enfermería está expuesto durante la jornada de trabajo, la exposición a agentes químicos y la constante incorporación de nuevas tecnologías, conocimientos y responsabilidades, se traduce en un ambiente hostil precursor de SB (Araya, Mantuliz, \& Parada, 2012). En Ecuador existen investigaciones que analizan los diferentes factores de riesgo psicosocial, destacando un estudio realizado al personal de enfermería que labora en el área de emergencia de un Hospital público de la ciudad de Quito, realizado en el año 2018, donde se evidencia que los factores psicosociales relativos a la Carga de trabajo (48\%), Demanda Psicológica (43\%) y Participación/Supervisión (51\%) presentan un nivel de riesgo más elevado entre la muestra investigada.

Sin embargo, los estudios antes mencionados no abarcan particularmente el caso de los trabajadores de la unidad de HD, que laboran como cuidadores directos y que obedecen a consideraciones particulares por el tipo de paciente, a partir de lo cual se pretende identificar los factores psicosociales presentes, medir la significancia de sus dimensiones y correlacionar las variables socio-laborales con los riesgos psicosociales existentes. En base a la problemática tratada anteriormente y su pertinencia, el objetivo de nuestro estudio pretende identificar y analizar aquellos factores de riesgo psicosocial en el ambiente laboral, en relación al nivel de riesgo presentado, ya que según Mingote (2011), hay un incremento notable del absentismo laboral y de patologías psicológicas, sobre todo en el área sanitaria, permitiendo tener un punto de referencia actual con respecto al problema.

Los salubristas o especialistas en Salud Pública se definen como aquellos que tienen como sujeto de salud las grandes comunidades o los grandes grupos (Martínez, 2018). Según De la Espriella (2015), la presencia de la salud ocupacional en una organización es de vital importancia ya que además de procurar el más alto bienestar físico, mental y social de los empleados, éste también busca establecer y sostener un medio ambiente de trabajo seguro y sano. Lahera (2002), sostiene que la relación entre trabajo y salud puede abordarse desde distintos ámbitos. Desde los riesgos que el
Vol. 3, Nro. 2, Publicado 2020-12-31

trabajo representa para ellos hasta lo que su percepción puede generar, y en este caso es mental.

Por tanto, hablar de riesgo laboral debe tomarse de una manera responsable y particularmente al contexto que se estudia, tal como afirma Chávez (2018) que los estudios de riesgo son necesariamente muy locales, porque dependen de condiciones específicas de cada sitio. Una peculiaridad de los estudios de riesgo es que lo que está expuesto, varía en el tiempo, tanto en cantidad, como en sus características; específicamente en los efectos del crecimiento demográfico y de la industrialización, que modifican e incrementan el riesgo, por lo que los estudios se vuelven rápidamente obsoletos.

Las dimensiones psicosociales según Moreno (2010) estudiadas en este contexto, se refieren a tres formas prevalentes, entre ellos: 1) factores psicosociales, 2) riesgo o factores psicosociales de estrés y 3) riesgos psicosociales. Aunque son términos próximos entre ellos, sus referencias históricas y conceptuales son diferentes, e incluso hay diferencias entre ellos que pueden ser notables. En el caso que nos atañe, trabajaremos con los Factores de Riesgo Psicosocial. El método de evaluación FPSICO de factores psicosociales es un instrumento de aplicación individualizada que permite realizar un diagnóstico de la situación de riesgo psicosocial de una empresa o de áreas parciales de la misma. Está elaborado por el Instituto Nacional de Seguridad e Higiene en el Trabajo del gobierno de España (INSHT). El cuestionario FPSICO versión 3.1 mide aquellas condiciones presentes en una situación laboral relativas a la organización y al contenido del trabajo, que pueden afectar tanto el desarrollo del mismo como la salud de los trabajadores (Instituto de Biomecánica de Valencia, 2019). La diálisis es un tratamiento que elimina las toxinas y los fluidos que sobran, producto de la condición crónica por la que padecen estos pacientes, para que no se acumulen en el cuerpo. Este proceso es un poco doloroso y traumático para los individuos que reciben este tratamiento, y también para los que ofrecen el servicio, por ende es necesario evaluar la salud mental de los colaboradores que presta este servicio y su impacto (Northwestern Medicine, 2016).

\section{Métodos:}

La metodología empleada en el este estudio es de tipo cuantitativa - descriptiva, correlacional, con corte transversal, cuyo fin reside en identificar los factores psicosociales y el nivel de riesgo al que está expuesto el personal salubrista que labora 
Periodo. Julio - Diciembre 2020

como cuidadores directos en las unidades de hemodiálisis de la ciudad de Portoviejo y Manta. La población se conformó por un total de 90 trabajadores de salud que laboran en tres unidades de diálisis en las ciudades de Portoviejo-Manta, cuyo personal está distribuido de la siguiente manera: Centro de diálisis Renacer con 30 trabajadores; Unidad de Hemodiálisis IESS-Manta con 32 trabajadores; Unidad de Hemodiálisis del hospital de especialidades "Portoviejo" con 28 profesionales de la salud, para un total de 90 trabajadores en los tres centros.

Se empleó un muestreo no probabilístico, puesto que fueron considerados todo el personal de atención de dichas unidades de HD, con base en los siguientes criterios de inclusión: Profesionales de la salud que atiendan directamente a los pacientes que asisten a los centros de diálisis, que lleven más de un año laborando en dichos centros y que se desempeñen como personal de salud (Médicos, enfermeras, auxiliares de enfermería, auxiliar de servicio y equipo multidisciplinario: Psicología, nutrición, trabajo social) a tiempo completo laborando en dichas áreas. Teniendo en cuenta estos criterios, la muestra seleccionada fue de 68 trabajadores, de los cuales se obtuvo la participación de 63 personas, debido a que 5 de ellos se encontraron de vacaciones en el momento de llevar a cabo las encuestas y entrevistas. Las profesiones objeto de estudio fueron: 16 médicos, 20 enfermeras y 27 profesionales pertenecientes al equipo multidisciplinario.

De igual forma, dentro del grupo de profesionales de la muestra estudiada, se realizó una entrevista donde se recolectaron datos básicos sobre las condiciones sociodemográficas y laborales, como las correspondientes al género, estado civil, cargo laboral, edad determinada por rangos (20-25 años, 26-30 años, 31-35 años, 36-40 años y mayores de 40 años) y antigüedad en el cargo (agrupadas de 1

\section{Resultados:}

Del total de la muestra estudiada, conformada por 63 profesionales sanitarios, vemos como descriptivos sociodemográficos una proporcionalidad respecto al género, $50.79 \%$ conformada por hombres y $49.21 \%$ por mujeres, el rango de edad que predomina es el comprendido entre 31 a 35 años $(34,92 \%)$. Con respecto al estado civil, el $49,21 \%$ son casados, seguido de los

\section{Vol. 3, Nro. 2, Publicado 2020-12-31}

a 3 años y mayores de 3 años). Respecto a la recolección de datos, para esta investigación se empleó como instrumento de medición el cuestionario Fpsico 3.1, validado y ampliamente utilizado en otros estudios nacionales e internacionales para conocer la problemática tratada, y se aplicó a la realidad de las instituciones escogidas.

Este instrumento consta de 44 preguntas, algunas múltiples, de forma que el número de ítems asciende a 89; ofrece información sobre 9 dimensiones o factores cuyo empleo nos permitió identificar y evaluar los diferentes factores de riesgo psicosocial, donde se compararon las variables por dimensión y cargo laboral (Instituto de Biomecánica de Valencia, 2019). Las dimensiones de riesgo psicosocial que valora este instrumento son: Tiempo de Trabajo (TT), Autonomía (AU), Carga de Trabajo (CT), Demandas Psicológicas (DP), Variedad/ Contenido (VC), Participación/ Supervisión (PS), Interés por el Trabajador/ Compensación (ITC), Desempeño de Rol (DR), Relaciones y Apoyo Social (RAS).

Una vez sistematizados los datos, se procedió a su análisis utilizando el Paquete Estadístico para Ciencias Sociales (SPSS) versión 22, a partir del cual se obtuvo los estadísticos descriptivos, frecuencia, tablas cruzadas (chi cuadrado de Pearson), pruebas de significancia para una muestra, correlaciones entre las variables sociodemográficas-laborales y las dimensiones psicosociales, definidas en este estudio como instrumento de medición; se agruparon dentro de cada dimensión aquellas que tienen un riesgo elevado o muy elevado ( $\mathrm{Si}$ ) y las que tienen una cuantificación de adecuado o moderado riesgo (No), categorizados para establecer relación entre las variables y poder estimar riesgos (ODDs Ratio)

solteros con $23,81 \%$. En relación a las profesiones, el $31.7 \%$ corresponden a enfermeros, el $25.4 \%$ a médicos, el $15.9 \%$ a equipo multidisciplinario, el $14.3 \%$ auxiliares de servicios y el $12.7 \%$ auxiliares de enfermería; el $78 \%$ tienen una antigüedad en el cargo de entre 1-3 años y el $22 \%$ restante mayor de 3 años. 
Tabla \# 1:

Factores de Riesgo Psicosocial, calificación general/ Nivel de riesgo

\begin{tabular}{|c|c|c|c|c|}
\hline Factores de Riesgo & Adecuada & Moderado & Elevado & $\begin{array}{c}\text { Muy } \\
\text { Elevado }\end{array}$ \\
\hline Tiempo de Trabajo (TT) & $59(93,65 \%)$ & $3(4,76 \%)$ & $1(1,59 \%)$ & - \\
\hline Autonomía (AU) & $61(96,83 \%)$ & $2(3,17 \%)$ & - & - \\
\hline Carga de trabajo (CT) & $38(60,32 \%)$ & $12(19.05 \%)$ & $9(14.2 \%)$ & $4(6.35 \%)$ \\
\hline $\begin{array}{l}\text { Demandas Psicológicas } \\
\text { (DP) }\end{array}$ & $30(47.62 \%)$ & $15(23.81 \%)$ & $12(19.1 \%)$ & $6(9.52 \%)$ \\
\hline $\begin{array}{l}\text { Variedad/ } \\
\text { Contenido (VC) }\end{array}$ & $59(93.65 \%)$ & $3(4.76 \%)$ & $1(1.59 \%)$ & - \\
\hline $\begin{array}{l}\text { Participación / } \\
\text { Supervisión (PS) }\end{array}$ & $43(68.25 \%)$ & $12(19.05 \%)$ & $6(9.52 \%)$ & $2(3.27 \%)$ \\
\hline $\begin{array}{l}\text { Interés por el Trabajador } \\
\text { Compensación } \\
\text { (ITC) }\end{array}$ & $36(57.15 \%)$ & $12(19.05 \%)$ & $9(14.3 \%)$ & $6(9.52 \%)$ \\
\hline Desempeño de Rol (DR) & $56(88.89 \%)$ & $5(7.94 \%)$ & $2(3.17 \%)$ & - \\
\hline $\begin{array}{l}\text { Relaciones y Apoyo } \\
\text { Social (RAS) }\end{array}$ & $59(93.65 \%)$ & $4(6.35 \%)$ & - & - \\
\hline
\end{tabular}

Revisando el nivel de Riesgo Psicosocial en términos generales, se encontró que, en el personal salubrista de $\mathrm{HD}$, el $89 \%$ presenta un riesgo adecuado-moderado (sin riesgo) frente al $11 \%$ (con riesgo) que evidencia un riesgo elevado o muy elevado. Sin embargo, al observar los resultados de acuerdo a las dimensiones de riesgo psicosocial, se encontró que las dimensiones que presentan un nivel mayor de riesgo son: Carga de Trabajo (20,5\%), Demandas Psicológicas (28,6\%), Interés por el Trabajador/Compensación (23,8\%); las dimensiones con un nivel de riesgo bajo son Autonomía y Relaciones / Apoyo Social (ver tabla 1). Para encontrar la relación entre las dimensiones de riesgo psicosocial y las variables dependientes socio-laborales, se recodificaron los niveles de los factores de riesgo psicosocial, agrupándolos concernientes a "elevado" y "muy elevado" como "con riesgo", contra los correspondientes a "adecuado" y "moderado" como "no riesgo".

Se encontró relación entre las dimensiones de riesgo psicosocial y el cargo laboral que desempeñan los salubristas en dos de las nueve dimensiones que evalúa el instrumento, presentando un nivel de riesgo mayor en los enfermeros comparados con los médicos, auxiliares y equipo multidisciplinario; así, en la carga de trabajo (CT), con un nivel de significancia (chi-cuadrado) de asociación positiva $(\mathrm{p}=0,00)$, presentan un riesgo del $55 \%$ para dicho factor, de igual manera en las demandas psicológicas $(\mathrm{p}=$ 0,00 ) con un riesgo del $70 \%$ (ver tabla 2). 
Tabla \# 2:

Vol. 3, Nro. 2, Publicado 2020-12-31

Asociación entre dimensiones de riesgo psicosocial, cargo laboral, antigüedad, edad y sexo

\begin{tabular}{|c|c|c|c|c|c|c|c|c|c|c|c|c|c|c|c|c|c|c|}
\hline \multicolumn{19}{|c|}{ Dimensiones del Riesgo Psicosocial } \\
\hline & \multicolumn{2}{|c|}{ TT (\%) } & \multicolumn{2}{|c|}{ AU (\%) } & \multicolumn{2}{|c|}{ CT (\%) } & \multicolumn{2}{|c|}{$\begin{array}{c}\text { DP } \\
(\%)\end{array}$} & \multicolumn{2}{|c|}{ VC (\%) } & \multicolumn{2}{|c|}{ PS (\%) } & \multicolumn{2}{|c|}{$\begin{array}{l}\text { ITC } \\
(\%)\end{array}$} & \multicolumn{2}{|c|}{$\begin{array}{c}\text { DR } \\
(\%)\end{array}$} & \multicolumn{2}{|c|}{ RAS } \\
\hline Cargos & $\begin{array}{l}\mathbf{N} \\
\mathbf{O}\end{array}$ & $\begin{array}{l}\text { val } \\
\text { or } \\
p\end{array}$ & No & $\begin{array}{c}\text { val } \\
\text { or } \\
\text { p }\end{array}$ & $\begin{array}{l}\mathbf{N} \\
\mathbf{O}\end{array}$ & $\begin{array}{c}\text { val } \\
\text { or } \\
\text { p }\end{array}$ & $\begin{array}{l}\mathbf{N} \\
\mathbf{O}\end{array}$ & $\begin{array}{l}\text { val } \\
\text { or } \\
\mathbf{p}\end{array}$ & $\begin{array}{l}\mathbf{N} \\
\mathbf{0}\end{array}$ & $\begin{array}{c}\text { val } \\
\text { or } \\
\text { p }\end{array}$ & $\mathbf{N}$ & $\begin{array}{c}\text { val } \\
\text { or } \\
\mathbf{p} \\
\end{array}$ & $\begin{array}{l}\mathbf{N} \\
\mathbf{O}\end{array}$ & $\begin{array}{l}\text { val } \\
\text { or } \\
\text { p }\end{array}$ & $\begin{array}{l}\mathbf{N} \\
\mathbf{O}\end{array}$ & $\begin{array}{l}\text { val } \\
\text { or } \\
\mathbf{p}\end{array}$ & $\begin{array}{l}\mathbf{N} \\
\mathbf{O}\end{array}$ & $\begin{array}{c}\text { val } \\
\text { or } \\
\mathbf{p}\end{array}$ \\
\hline Médicos & 94 & \multirow{5}{*}{$\begin{array}{l}0.5 \\
82\end{array}$} & 100 & \multirow{5}{*}{$\begin{array}{l}0.1 \\
42\end{array}$} & 94 & \multirow{5}{*}{$\begin{array}{c}0.0 \\
01\end{array}$} & $\begin{array}{c}10 \\
0\end{array}$ & \multirow{5}{*}{$\begin{array}{l}0.0 \\
01\end{array}$} & 94 & \multirow{5}{*}{$\begin{array}{l}0.5 \\
82\end{array}$} & 88 & \multirow{5}{*}{$\begin{array}{l}0.5 \\
60\end{array}$} & 81 & \multirow{5}{*}{$\begin{array}{l}0.3 \\
42\end{array}$} & 94 & \multirow{5}{*}{$\begin{array}{l}0.3 \\
07\end{array}$} & $\begin{array}{c}10 \\
0\end{array}$ & \multirow{5}{*}{$\begin{array}{l}0.5 \\
27\end{array}$} \\
\hline $\begin{array}{l}\text { Enferme } \\
\text { ros }\end{array}$ & $\begin{array}{c}10 \\
0 \\
\end{array}$ & & 100 & & 45 & & 30 & & $\begin{array}{c}10 \\
0\end{array}$ & & 90 & & 75 & & \begin{tabular}{|c|}
10 \\
0 \\
\end{tabular} & & $\begin{array}{c}10 \\
0\end{array}$ & \\
\hline $\begin{array}{l}\text { Aux. de } \\
\text { Servicio } \\
\text { s }\end{array}$ & $\begin{array}{c}10 \\
0\end{array}$ & & 100 & & $\begin{array}{c}10 \\
0\end{array}$ & & 78 & & 89 & & 78 & & 55 & & 89 & & $\begin{array}{c}10 \\
0\end{array}$ & \\
\hline $\begin{array}{l}\text { Aux. de } \\
\text { Enferme } \\
\text { ría }\end{array}$ & $\begin{array}{c}10 \\
0\end{array}$ & & 100 & & $\begin{array}{l}87 \\
.5\end{array}$ & & $\begin{array}{r}87 \\
.5\end{array}$ & & $\begin{array}{r}87 \\
.5\end{array}$ & & 87 & & 87 & & $\begin{array}{c}10 \\
0\end{array}$ & & $\begin{array}{c}10 \\
0\end{array}$ & \\
\hline Otros & $\begin{array}{c}10 \\
0\end{array}$ & & 100 & & $\begin{array}{c}10 \\
0\end{array}$ & & $\begin{array}{c}10 \\
0 \\
\end{array}$ & & $\begin{array}{c}10 \\
0\end{array}$ & & 90 & & 80 & & \begin{tabular}{|c|}
10 \\
0 \\
\end{tabular} & & $\begin{array}{c}10 \\
0\end{array}$ & \\
\hline $\begin{array}{l}\text { Antigü } \\
\text { edad }\end{array}$ & $\begin{array}{l}\mathbf{N} \\
\mathbf{0}\end{array}$ & $\begin{array}{c}\text { val } \\
\text { or } \\
p\end{array}$ & No & $\begin{array}{c}\text { val } \\
\text { or } \\
\text { p }\end{array}$ & $\begin{array}{l}\mathbf{N} \\
\mathbf{O}\end{array}$ & $\begin{array}{c}\text { val } \\
\text { or } \\
p\end{array}$ & $\begin{array}{l}\mathbf{N} \\
\mathbf{O}\end{array}$ & $\begin{array}{c}\text { val } \\
\text { or } \\
p\end{array}$ & $\begin{array}{l}\mathbf{N} \\
\mathbf{0}\end{array}$ & $\begin{array}{c}\text { val } \\
\text { or } \\
\text { p }\end{array}$ & $\begin{array}{l}\mathbf{N} \\
\mathbf{0}\end{array}$ & $\begin{array}{c}\text { val } \\
\text { or } \\
p\end{array}$ & $\begin{array}{l}\mathbf{N} \\
\mathbf{0}\end{array}$ & $\begin{array}{c}\text { val } \\
\text { or } \\
\mathbf{p}\end{array}$ & $\begin{array}{l}\mathbf{N} \\
\mathbf{0}\end{array}$ & $\begin{array}{c}\text { val } \\
\text { or } \\
\mathbf{p}\end{array}$ & $\begin{array}{l}\mathbf{N} \\
\mathbf{0}\end{array}$ & $\begin{array}{c}\text { val } \\
\text { or } \\
p\end{array}$ \\
\hline $\begin{array}{l}1-3 \\
\text { años }\end{array}$ & 94 & \multirow{2}{*}{$\begin{array}{c}0.0 \\
59\end{array}$} & 100 & \multirow[b]{2}{*}{ a } & 84 & \multirow{2}{*}{$\begin{array}{l}0.1 \\
14\end{array}$} & 74 & \multirow{2}{*}{$\begin{array}{l}0.5 \\
02\end{array}$} & $\begin{array}{c}10 \\
0 \\
\end{array}$ & \multirow{2}{*}{$\begin{array}{l}0.0 \\
59\end{array}$} & 96 & \multirow{2}{*}{$\begin{array}{l}0.0 \\
00\end{array}$} & 84 & \multirow{2}{*}{$\begin{array}{l}0.0 \\
09\end{array}$} & \begin{tabular}{|c|}
10 \\
0 \\
\end{tabular} & \multirow{2}{*}{$\begin{array}{l}0.0 \\
07\end{array}$} & $\begin{array}{c}10 \\
0 \\
\end{array}$ & \multirow{2}{*}{$a$} \\
\hline $\begin{array}{l}>3 \\
\text { años }\end{array}$ & 92 & & 100 & & 14 & & 64 & & 93 & & 57 & & 50 & & 86 & & $\begin{array}{c}10 \\
0 \\
\end{array}$ & \\
\hline $\begin{array}{l}\text { Edad } \\
\text { (años) }\end{array}$ & $\begin{array}{l}\mathbf{N} \\
\mathbf{0}\end{array}$ & $\begin{array}{l}\text { val } \\
\text { or } \\
p\end{array}$ & No & $\begin{array}{c}\text { val } \\
\text { or } \\
p\end{array}$ & $\begin{array}{l}\mathbf{N} \\
\mathbf{O}\end{array}$ & $\begin{array}{c}\text { val } \\
\text { or } \\
\text { p }\end{array}$ & $\begin{array}{l}\mathbf{N} \\
\mathbf{O}\end{array}$ & $\begin{array}{c}\text { val } \\
\text { or } \\
\mathbf{p}\end{array}$ & $\begin{array}{l}\mathbf{N} \\
\mathbf{0}\end{array}$ & $\begin{array}{c}\text { val } \\
\text { or } \\
\text { p }\end{array}$ & $\begin{array}{l}\mathbf{N} \\
\mathbf{0}\end{array}$ & $\begin{array}{c}\text { val } \\
\text { or } \\
p\end{array}$ & $\begin{array}{l}\mathbf{N} \\
\mathbf{0}\end{array}$ & $\begin{array}{c}\text { val } \\
\text { or } \\
\text { p }\end{array}$ & $\begin{array}{l}\mathbf{N} \\
\mathbf{0}\end{array}$ & $\begin{array}{c}\text { val } \\
\text { or } \\
\mathbf{p}\end{array}$ & $\begin{array}{c}\mathbf{N} \\
\mathbf{O}\end{array}$ & $\begin{array}{l}\text { val } \\
\text { or } \\
p\end{array}$ \\
\hline 20 & $\begin{array}{l}3 . \\
2\end{array}$ & \multirow{5}{*}{$\begin{array}{l}0.4 \\
69\end{array}$} & 3.2 & \multirow{5}{*}{ a } & $\begin{array}{l}3 . \\
2\end{array}$ & & $\begin{array}{l}3 . \\
2\end{array}$ & & $\begin{array}{l}3 . \\
2\end{array}$ & & & & $\begin{array}{l}1 . \\
6\end{array}$ & & $\begin{array}{l}3 . \\
2\end{array}$ & & $\begin{array}{l}3 . \\
2\end{array}$ & \\
\hline 26 a 30 & \begin{tabular}{|c|}
19 \\
.0
\end{tabular} & & $\begin{array}{c}19 . \\
0\end{array}$ & & $\begin{array}{l}15 \\
.9\end{array}$ & & $\begin{array}{l}14 \\
.3\end{array}$ & & $\begin{array}{l}19 \\
.0\end{array}$ & & 19 & & $\begin{array}{l}14 \\
.3\end{array}$ & & \begin{tabular}{|l|}
19 \\
.0
\end{tabular} & & $\begin{array}{l}19 \\
.0\end{array}$ & \\
\hline 31 a 35 & $\begin{array}{l}34 \\
.9\end{array}$ & & $\begin{array}{c}34 . \\
9\end{array}$ & & $\begin{array}{l}25 \\
.4\end{array}$ & $\begin{array}{c}0.8 \\
05\end{array}$ & $\begin{array}{l}25 \\
.4\end{array}$ & $\begin{array}{l}0.8 \\
24\end{array}$ & $\begin{array}{l}34 \\
.9\end{array}$ & $\begin{array}{l}0.4 \\
69\end{array}$ & $\begin{array}{l}30 \\
.2\end{array}$ & $\begin{array}{l}0.3 \\
56\end{array}$ & $\begin{array}{l}27 \\
.0\end{array}$ & $\begin{array}{l}0.0 \\
68\end{array}$ & $\begin{array}{r}33 \\
.3\end{array}$ & $\begin{array}{l}0.7 \\
84\end{array}$ & $\begin{array}{c}34 \\
.9\end{array}$ & a \\
\hline 36 a 40 & $\begin{array}{l}20 \\
.6\end{array}$ & & $\begin{array}{c}20 . \\
6\end{array}$ & & $\begin{array}{l}19 \\
.0\end{array}$ & & $\begin{array}{l}15 \\
.9\end{array}$ & & $\begin{array}{l}20 \\
.6\end{array}$ & & 10 & & $\begin{array}{l}22 \\
.2\end{array}$ & & $\begin{array}{l}20 \\
.6\end{array}$ & & $\begin{array}{l}22 \\
.2\end{array}$ & \\
\hline$>$ a 40 & \begin{tabular}{|l|}
20 \\
.6 \\
\end{tabular} & & $\begin{array}{c}20 . \\
6\end{array}$ & & $\begin{array}{l}15 \\
.9 \\
\end{array}$ & & $\begin{array}{l}12 \\
.7 \\
\end{array}$ & & $\begin{array}{l}20 \\
.6 \\
\end{array}$ & & 17 & & \begin{tabular}{|l|}
11 \\
.1 \\
\end{tabular} & & \begin{tabular}{|l|}
20 \\
.6 \\
\end{tabular} & & $\begin{array}{l}20 \\
.6 \\
\end{array}$ & \\
\hline Sexo & $\begin{array}{l}\mathbf{N} \\
\mathbf{O}\end{array}$ & $\begin{array}{l}\text { val } \\
\text { or } \\
p\end{array}$ & No & $\begin{array}{c}\text { val } \\
\text { or } \\
\text { p }\end{array}$ & $\begin{array}{l}\mathbf{N} \\
\mathbf{O}\end{array}$ & $\begin{array}{c}\text { val } \\
\text { or } \\
p\end{array}$ & $\begin{array}{l}\mathbf{N} \\
\mathbf{O}\end{array}$ & $\begin{array}{c}\text { val } \\
\text { or } \\
p\end{array}$ & $\begin{array}{l}\mathbf{N} \\
\mathbf{0}\end{array}$ & $\begin{array}{c}\text { val } \\
\text { or } \\
\text { p }\end{array}$ & $\begin{array}{l}\mathbf{N} \\
\mathbf{0}\end{array}$ & $\begin{array}{c}\text { val } \\
\text { or } \\
p\end{array}$ & $\begin{array}{l}\mathbf{N} \\
\mathbf{O}\end{array}$ & $\begin{array}{c}\text { val } \\
\text { or } \\
p\end{array}$ & $\begin{array}{l}\mathbf{N} \\
\mathbf{O}\end{array}$ & $\begin{array}{c}\text { val } \\
\text { or } \\
\mathbf{p}\end{array}$ & $\begin{array}{l}\mathbf{N} \\
\mathbf{O}\end{array}$ & $\begin{array}{l}\text { val } \\
\text { or } \\
\text { p }\end{array}$ \\
\hline $\begin{array}{l}\text { Masculi } \\
\text { no }\end{array}$ & 93 & 0.3 & $\begin{array}{c}94 . \\
2\end{array}$ & & $\begin{array}{l}34 \\
.9 \\
\end{array}$ & 0.1 & $\begin{array}{l}30 \\
.2 \\
\end{array}$ & 0.5 & $\begin{array}{l}49 \\
.2\end{array}$ & 0.3 & $\begin{array}{l}44 \\
.4\end{array}$ & 0.4 & \begin{tabular}{|c|}
38 \\
.1 \\
\end{tabular} & 0.4 & \begin{tabular}{|l|}
47 \\
.6 \\
\end{tabular} & 0.0 & $\begin{array}{c}49 \\
.2 \\
\end{array}$ & \\
\hline $\begin{array}{l}\text { Femeni } \\
\text { no }\end{array}$ & $\begin{array}{l}3 . \\
0\end{array}$ & 21 & $\begin{array}{c}54 . \\
8\end{array}$ & 8 & $\begin{array}{l}44 \\
.4 \\
\end{array}$ & 05 & $\begin{array}{c}41 \\
.3 \\
\end{array}$ & 02 & $\begin{array}{l}49 \\
.2\end{array}$ & 21 & $\begin{array}{r}42 \\
.9 \\
\end{array}$ & 78 & $\begin{array}{c}38 \\
.1\end{array}$ & 82 & $\begin{array}{l}47 \\
.6\end{array}$ & 07 & $\begin{array}{c}50 \\
.8\end{array}$ & 8 \\
\hline
\end{tabular}


Periodo. Julio - Diciembre 2020

Tabla \# 3:

Vol. 3, Nro. 2, Publicado 2020-12-31

Estimación de riesgo

\begin{tabular}{|c|c|c|c|c|c|c|c|c|c|}
\hline & \multicolumn{9}{|c|}{ ODDS RATIO (OR) } \\
\hline & TT & AU & CT & DP & VC & PS & ITC & DR & $\begin{array}{c}\text { RA } \\
\text { S }\end{array}$ \\
\hline Cargos & \multicolumn{9}{|c|}{ IC: $95 \%$} \\
\hline Médico & - & - & - & - & - & - & - & - & - \\
\hline $\begin{array}{l}\text { Enferme } \\
\text { ro }\end{array}$ & - & - & $\begin{array}{l}0,478- \\
27,110 \\
(3.600)\end{array}$ & $\begin{array}{c}0,102- \\
6,249 \\
(0,800)\end{array}$ & - & $\begin{array}{c}0,200- \\
7,404 \\
(0,385)\end{array}$ & $\begin{array}{l}0,174- \\
22,949 \\
(2.000)\end{array}$ & - & - \\
\hline $\begin{array}{l}\text { Aux. de } \\
\text { servicios }\end{array}$ & - & - & Then & $\begin{array}{c}0,016- \\
10,017 \\
(0,400)\end{array}$ & - & $\begin{array}{l}0,100- \\
62,605 \\
(2,500)\end{array}$ & $\begin{array}{c}0,280- \\
8,952 \\
(0.500)\end{array}$ & - & - \\
\hline $\begin{array}{l}\text { Aux. } \\
\text { enferme } \\
\text { ría }\end{array}$ & - & - & $\begin{array}{c}0,992- \\
5,489 \\
(2.333)\end{array}$ & $\begin{array}{c}0,992- \\
5,489 \\
(2,333)\end{array}$ & - & $\begin{array}{c}0,992- \\
5,489 \\
(2,333)\end{array}$ & $\begin{array}{c}0,992- \\
5,489 \\
(2.333)\end{array}$ & - & - \\
\hline Otros & - & - & - & - & - & - & - & - & - \\
\hline \multicolumn{10}{|l|}{$\begin{array}{l}\text { Antigüe } \\
\text { dad }\end{array}$} \\
\hline $\begin{array}{l}1 \text { a } 3 \\
\text { años }\end{array}$ & - & - & $\begin{array}{c}0,479- \\
1,176 \\
(0,750)\end{array}$ & $\begin{array}{c}0,655- \\
1,245 \\
(0.903) \\
\end{array}$ & - & $\begin{array}{c}0,080- \\
0,976 \\
(0,293)\end{array}$ & $\begin{array}{c}0,383- \\
1,017 \\
(0,624)\end{array}$ & - & - \\
\hline$>3$ años & $\begin{array}{c}2,942- \\
7,733 \\
(4.769)\end{array}$ & - & $\begin{array}{c}0,863- \\
5,293 \\
(2,137)\end{array}$ & $\begin{array}{c}0,539- \\
3,580 \\
(1.389)\end{array}$ & $\begin{array}{c}2,942- \\
7,733 \\
(4.769)\end{array}$ & $\begin{array}{c}2,423- \\
10,973 \\
(5,156)\end{array}$ & $\begin{array}{c}1,337- \\
7,658 \\
(3,200)\end{array}$ & $\begin{array}{c}3,061- \\
8,441 \\
(5,083)\end{array}$ & - \\
\hline \multicolumn{10}{|l|}{ Sexo } \\
\hline $\begin{array}{l}\text { Masculin } \\
0\end{array}$ & $\begin{array}{c}1,559- \\
2,565 \\
(2,000)\end{array}$ & - & $\begin{array}{c}0,234- \\
1,288 \\
(0,549)\end{array}$ & $\begin{array}{c}0,287- \\
1,161 \\
(0,577)\end{array}$ & $\begin{array}{c}1,559- \\
2,565 \\
(2,000)\end{array}$ & $\begin{array}{c}0,698- \\
2,321 \\
(1,273)\end{array}$ & $\begin{array}{c}0,615- \\
1,852 \\
(1,067)\end{array}$ & $\begin{array}{c}0,241- \\
4,021 \\
(0,984)\end{array}$ & - \\
\hline $\begin{array}{l}\text { Femenin } \\
\text { o }\end{array}$ & - & - & $\begin{array}{c}0,975- \\
2,539 \\
(1,573)\end{array}$ & $\begin{array}{c}0,984- \\
2,533 \\
(1,579)\end{array}$ & - & $\begin{array}{c}0,290- \\
1,870 \\
(0,737)\end{array}$ & $\begin{array}{c}0,507- \\
1,719 \\
(0,933)\end{array}$ & $\begin{array}{c}0,248- \\
4,161 \\
(1,017)\end{array}$ & - \\
\hline
\end{tabular}

De igual manera, la dimensión de Participación y Supervisión, se comportó como factor de riesgo en auxiliares de enfermería y auxiliares de servicio cuando se compara con los otros cargos, (O.R 2.33 I.C. al 95\%) (OR $2.50 \mathrm{IC}$ al 95\%) respectivamente (ver tabla 3). Respecto a la antigüedad del cargo, las dimensiones referentes al Contenido del Trabajo (OR 2.13 IC al 95\%), Participación y Supervisión (OR 5.15 IC al 95\%), Tiempo de Discusión:

En el presente estudio se identificaron como dimensiones con un nivel de riesgo elevado a las demandas psicológicas $(70 \%)$ y a la carga de trabajo (55\%), siendo los enfermeros el grupo más vulnerable entre los profesionales de presentar niveles elevados de riesgo psicosocial.
Trabajo (OR 4.76 IC al 95\%), Variedad/Contenido (OR 4.76) e Interés por el Trabajador /Compensación (OR 3.20 IC al 95\%), presentan una estimación de riesgo mayor y significativa en el personal que labora más de 3 años en sus instituciones con respecto al trabajador cuya antigüedad en su cargo está entre 1 a 3 años (ver tabla 3).

Este resultado concuerda con algunas investigaciones que relacionan de manera significativa la demanda psicológica (dp) como principal factor de riesgo en el grupo de los enfermeros.

Asimismo, un estudio realizado en un hospital público de la ciudad de quito, reportó un nivel de riego muy elevado en dimensiones carga de trabajo, demandas psicológicas y tiempo de trabajo 
en el personal de salud que labora en el área de emergencia, coincidiendo en dos dimensiones con el nivel de riesgo presentado en nuestra investigación. En Colombia, se realizó una investigación por Sarsosa, Charria, \& arenas (2014) sobre caracterización de los riesgos psicosociales intralaborales en jefes asistenciales de cinco clínicas nivel iii de Santiago de Cali. De esta investigación coincidimos en la demanda de trabajo como factor significativo de riesgo para los profesionales de la salud.

Los estudios coinciden que la salud mental de los trabajadores salubristas de centros de salud influye directamente en el trabajo realizado a los clientes. Existe una relación importante entre los estudios realizados en otros países sobre la salud mental de los profesionales salubristas y la atención brindada a los usuarios. En estos estudios también se identificaron significancias en el ambiente de trabajo y en posibilidades de desarrollo, aunque no fueron parte de nuestro campo de trabajo en las unidades seleccionadas.

No obstante, en nuestra investigación se identificó a un grupo de trabajadores con una estimación de riesgo significativa y elevada, a comparación de los otros cargos laborales (auxiliares de enfermería y auxiliares de servicio).

\section{Conclusiones:}

En general, los riesgos más importantes para los salubristas se basan en los denominados: carga de trabajo, demanda psicológica, participación supervisión y el interés por el trabajador o su compensación. La antigüedad en el cargo se relaciona de manera directa e influye con la presencia de riesgo en los factores de carga de trabajo (sobrecarga laboral), participación / supervisión y desempeño del rol.
ISSN 2773-7705

Periodo. Julio - Diciembre 2020

\section{Vol. 3, Nro. 2, Publicado 2020-12-31}

Con respecto al sexo y los grupos de edad, no se evidenció una relación directa, ni posee mayor relevancia en este estudio en comparación con las dimensiones psicosociales.

Los auxiliares son los que mayor probabilidad de riesgo presentan en participación/ supervisión (auxiliares de servicios), itc, dp y en ct (auxiliares de enfermería) dentro de dicho grupo de colaboradores, comparado con los médicos, enfermeros y equipo multidisciplinario; por lo tanto, nos permite orientar las intervenciones que deberían hacerse en ellos, una investigación específica de estos riesgos a fin de identificar los aspectos donde se encuentren las fallas organizacionales, e implementar programas de prevención sobre los factores psicosociales presentes entre los trabajadores, enfocados en las demandas psicológicas y la carga de trabajo, cuyo nivel de riesgo es alto entre los grupos de profesionales mayormente afectados.

El principal aporte de este trabajo se concentra en poder mostrar los factores de riesgos psicosociales a los que están expuestos el personal salubrista que labora en unidades de hd públicas y privadas, cuyas implicaciones son importantes tanto para el trabajador como para los usuarios, ya que en dicho grupo de profesionales se requiere que se encuentren en excelentes condiciones mentales para poder otorgar cuidados directos de alta calidad a los pacientes; además, se pudo relacionar los diferentes niveles de riesgos psicosociales con respecto a cargos, antigüedad, edad y sexo. Las limitaciones de esta investigación radicaron en que se consideró solo tres centros de diálisis, cuya selección dependió de la autorización de sus administradores en el tiempo del estudio; por aquello, de igual manera, no permitió profundizar el análisis sobre el impacto en la salud mental.

\section{Bibliografía:}

Araya, Silvia Barrios; Mantuliz, Ma Cecilia Arechabala: Parada, Victoria Valenzuela (2012). Relación entre carga laboral y burnout en enfermeras de unidades de diálisis. Enfermería Nefrológica.

Castro, N. (2018). Riesgos Psicosociales y Salud Laboral en Centros de Salud. Obtenido de Universidad Arturo Prat, Facultad de Ciencias de la Salud, Psicólogo, Magíster en Dirección y Gestión de Recursos Humanos, Académico Carrera de Psicología. : https://scielo.conicyt.cl/pdf/cyt/v20n63/0718-2449-cyt-20-63-00155.pdf

Chávez, S. (2018). El concepto de riesgo. Obtenido de Recursos Naturales y Sociedad: https://doi.org/10.18846 renaysoc. 2018.04 .04 .01 .0003

De la Espriella, A. (2015). La importancia de la salud ocupacional en las organizaciones. Obtenido de https://blog.acsendo.com/la-importancia-de-la-salud-ocupacional-en-las-organizaciones 
ISSN 2773-7705

Periodo. Julio - Diciembre 2020

Vol. 3, Nro. 2, Publicado 2020-12-31

Flynn, Thomas-Hawkins, \& Clarke (2009). Organizational Traits, Care Processes, and Burnout Among Chronic Hemodialysis Nurses. West J Nurs Res.

Instituto de Biomecánica de Valencia. (2019). FPSICO (INSHT). Obtenido de http://ergodep.ibv.org/procedimientos/11-metodos-psicosociales-especificos/475-fpsico-insht.html

Lahera, M. (2002). Factores Psicosociales. Obtenido de https://w3.ual.es/GruposInv/Prevencion/evaluacion/procedimiento/F.\%20Factores\%20Psicosociales.pdf

Mandujano, A., \& Danae, J. (2018). Estrés Laboral en cuidadores formales de un Centro de Diálisis y cuidadores informales de la ciudad de Quito durante el año 2017. DSpace.

Martínez, A. (2018). Asociación Uruguaya de Salubristas. Obtenido de Fue creada una nueva Sociedad Cientifica: https://www.smu.org.uy/publicaciones/noticias/noticias91/salubris.htm

Mingote, J. (2011). Programa de Atención Integral al profesional sanitario enfermo (UVOPSE_PAIPSE). Consejería de Sanidad. Comunidad Autónoma de Madrid. Madrid. España. Obtenido de http://scielo.isciii.es/scielo.php?script=sci_arttext\&pid=S0465-546X2011000500012

Moreno. (2010). Factores y riesgos, psicosociales, formas, consecuencias, medidas y buenas prácticas. Obtenido LOS RIESGOS PSICOSOCIALES: http://comisionnacional.insht.es/InshtWeb/Contenidos/Documentacion/PUBLICACIONES\%2OPROFESIONA LES/factores\%20riesgos\%20psico.pdf

MSP. (2018). Prevención, diagnóstico y tratamiento de la enfermedad renal crónica. Obtenido de https://www.salud.gob.ec/wp-

content/uploads/2018/10/guia_prevencion_diagnostico_tratamiento_enfermedad_renal_cronica_2018.pdf

Northwestern Medicine. (2016). Educación del paciente, cuidado y tratamiento. Obtenido de Hemodiálisis

OSHA. (2019). Agencia Europea para la Seguridad y la Salud en el Trabajo. Obtenido de Los riesgos psicosociales y el estrés en el trabajo: https://osha.europa.eu/es/themes/psychosocial-risks-and-stress

Rudnicki, T. (2006). Aspectos emocionales del paciente renal crónico. Sol de Invierno, 279-288. Obtenido de http://pepsic.bvsalud.org/scielo.php?script=sci_arttext\&pid=S1794-99982006000200008\&lng=pt\&tlng=es.

Sarsosa, Charria, \& Arenas. (2014). Caracterización de los riesgos psicosociales intralaborales en jefes asistenciales de cinco clínicas nivel III de Santiago de Cali (Colombia). Obtenido de http://www.scielo.org.co/pdf/rgps/v13n27/v13n27a21.pdf 